International Journal of Engineering \& Technology, $7(3.13)(2018) 55-60$
International Journal of Engineering \& Technology
SPC

\title{
Robust Diagnosis of Industrial Systems by Bond graph Model
}

\author{
Abderrahmene Sellami $^{1 *}$, Dhia Mzoughi², Abdelkader Mami ${ }^{3}$ \\ ${ }^{1-3}$ Analysis, Design and Control Systems Laboratory-ENIT, \\ Faculty of Sciences of Tunis, Tunis El Manar University, Tunis, Tunisia \\ *Corresponding author E-mail: abderrahmenesallami@gmail.com
}

\begin{abstract}
This paper aims to solve a research problem by a robust diagnosis of a hydraulic system with sand filter by approach of graph of connection using fractional linear transformations (BG-LFT). The method we develop is based on the use of analytic redundant relations by a bond graph graph model (BG-ARRs). These relationships not only allow the detection and isolation of defects on the various elements of the system, but also the location by structural and causal analysis. The results suggest that the use of the link diagram model for a valve fault (eg an $R_{I}$ valve is blocked), figures 9.a), 9.b) and 9.c) show that the residual models $r_{l}$ and $r_{2}$ become non-zero, so the flow and pressure levels are zero at reservoir $C_{2}$. These values mean that these residues are sensitive to the variation of the flow rate at the level of the valve $R_{l}$, which is confirmed by the theoretical results presented in table 2 . The simulation of the system is carried out by the software dedicated to the bond graph approach
\end{abstract}

Keywords: Robust Diagnosis, Bond Graph, Hydraulic System, Analytical Redundancy Relationships, Modelling and Simulations.

\section{Introduction}

Among the objectives of the automatic, one concerns the search for control means for physical systems often of different natures, electrical, mechanical, hydraulic and thermal, etc. [1, 2, 3 and 4]. System modeling is then the first step in the development of a control law. The model that describes physical reality is usually obtained from an idealized description of the system and only the dominant phenomena are often taken into account. In many cases, automation engineers use mathematical models that, in spite of their flexibility, quickly lose the physical meaning of the system and generally do not allow the model to be reverted to refine the modeling or improve the design of the system to simplify its control $[5,6,7,8,9$ and 10]. The multidisciplinary nature of the systems does not allow easy communication between experts in different fields. In addition, automation specialists are increasingly specializing in a particular field. There is a pronounced need for a modeling language to meet these needs in order to optimize this important modeling step. One of the tools that meet these criteria is the graph or graph of the links. Indeed, this language relies mainly on the notion of power transfer between the different parts or components of the system and on the transformation of energy in these components (dissipation, storage, conversion of the energy domain). These different phenomena, analogous in all physical domains, are graphically encoded [11, 12, 13, 14 and 15]. The unified nature of the jump chart is a universal language of communication between experts from different disciplines. Thanks to this decomposition and graphical representation, it is easy to break up the system into parts or subsystems and to return to any subsys- tem to improve its design or to take into account a neglected physical phenomenon taken into account. The bond graph model can then be considered as an intermediate model between the physical system and the associated mathematical model.

This document is organized in three sections as follows: Section 1 deals with the interest of the bond graph. In the first part of this article; we present the robust supervision system based on a bond graph and an external model. In the second part, the developed methodology is applied to a real hydraulic system. In this article, we will present the utility of the graphical linking tool for the supervision of industrial systems. In the first part, we will give the different approaches by using a jump graph for the design of a super vision system (qualitative and quantitative approach), the second part is dedicated to the determination of the bond graph approach in using the LFT (Fractional Linear Transformations) form to generate residuals and adaptive thresholds for normal operation with perfect separation.

\section{Interest of the Bond Graph}

Bond Graph-based modeling relies mainly on the concept of generalized stress and flux variables that allow the representation of balance sheets and energy exchanges between the different elements of a system [16, 17, 18 and 19]. In this approach, an energy exchange between two elements is represented by a half-arrow link indicating the direction of the transfer. These half-arrows are called "bonds", each is labeled by a force variable e and a flux variable $f$. The product of these two variables corresponds to the 
power "carried" by the leap. This power is counted positively in the direction of the half-arrow. The advantage of this modeling is that the choice of e and $\mathrm{f}$ depends only on the physical domain of the system to be represented, see figure 1 .

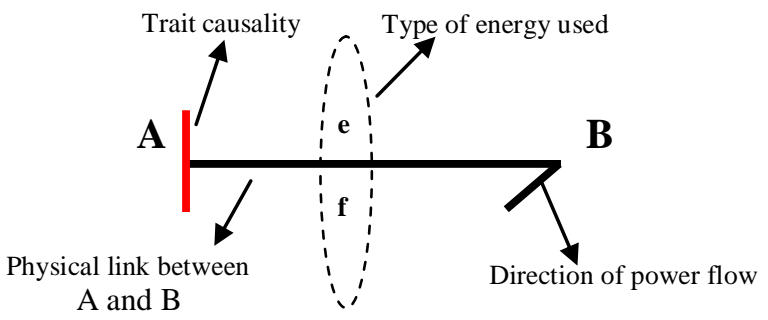

Fig.1: Representation of a physical system by bond graph

\section{Supervision of Industrial Systems Using Bond Graph}

The supervision of industrial systems using the bond graph approach is based on the generation of redundant analytical relationships [20, 21and 22].

This supervision system is represented according to the following figure 2 .

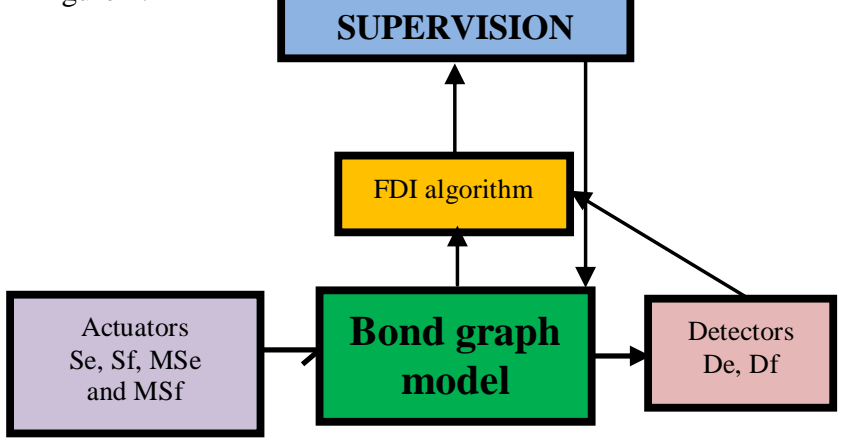

Fig. 2: Bond Graph representation of a monitoring system

The bond graph tool has three properties (structural, causal and multidisciplinary) and becomes very efficient for the realization of models in a generic way and also introduced for the contribution to the design of supervisory systems of industrial systems. Every industrial system includes:

- Set of actuators: there are two types, simple type modeled by sources $S e$ (effort) or $S f$ (flow) and type controlled modeled by $M S e$ (controlled effort) or $M S f$ (controlled flow).

- Set of sensors: they represent the information given by the industrial system; they are modeled by $D e$ (sensor effort) or $D f$ (sensor flow).

- Faults Detection and Isolation (FDI) Algorithms: They are algorithms that receive online sensor information and deliver alarms to the monitoring system in the event of faults that may have occurred in the course of time.

\section{Robust Diagnosis by Bond Graph}

\section{1. Bond Graph Model by LT Representation}

Fractional linear transformations (LFTs) are very efficient in determining the modeling of uncertain industrial systems to separate nominal residuals from uncertainties. These nominal values are grouped together under a matrix called $\mathrm{P}$, the other parametric uncertainties are combined in a structure diagonal matrix $\Delta$ shown in figure 3 [24, 25, 26, 27 and 28].

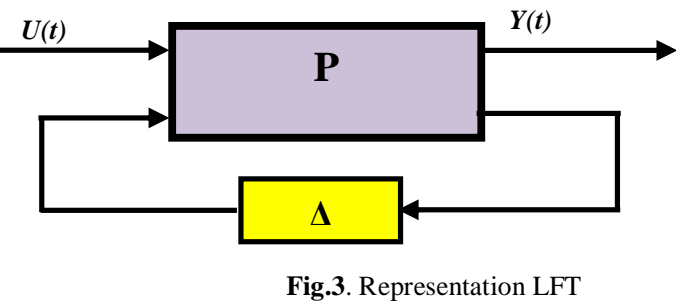

\section{2. Construction of a Model BG-LFT}

All industrial systems can be modeled by a bond graph model according to figure 3. Indeed, the input signal is modeled by a source of effort $(\mathrm{Se})$ or a source of the flow $(\mathrm{Sf})$, the whole system is modeled by resistive elements $(R)$ and storage elements ( $I$ or $C)$ while the detectors are modeled by elements of the detectors ( $D e$ or $D f$ ).
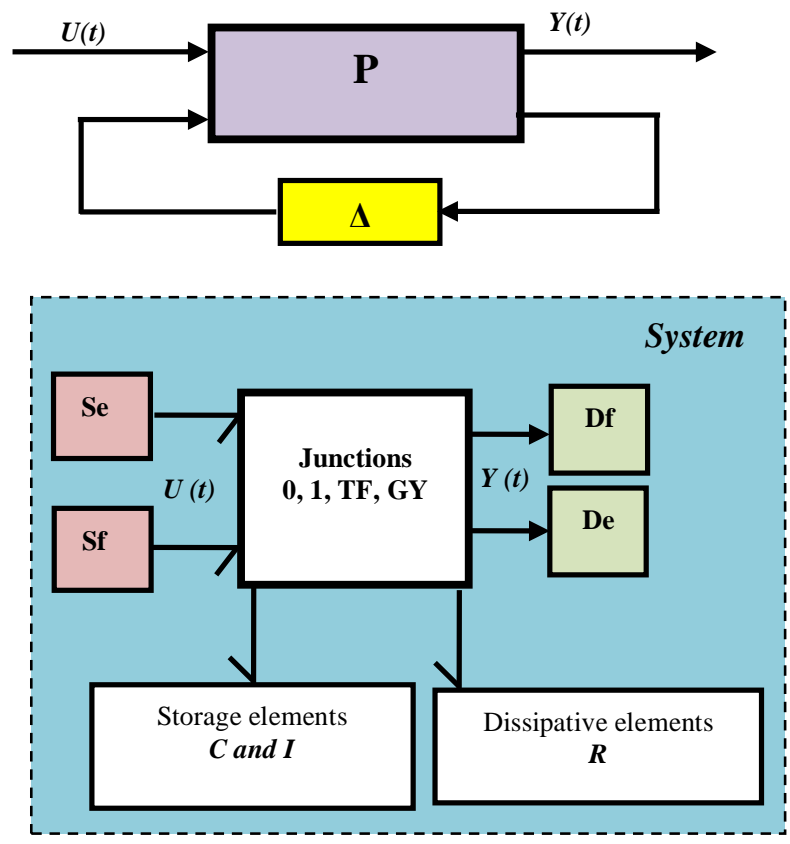

Fig.4. Industrial system described by bond graph

Full BG-LFT can then be represented by the diagram in figure 5 .

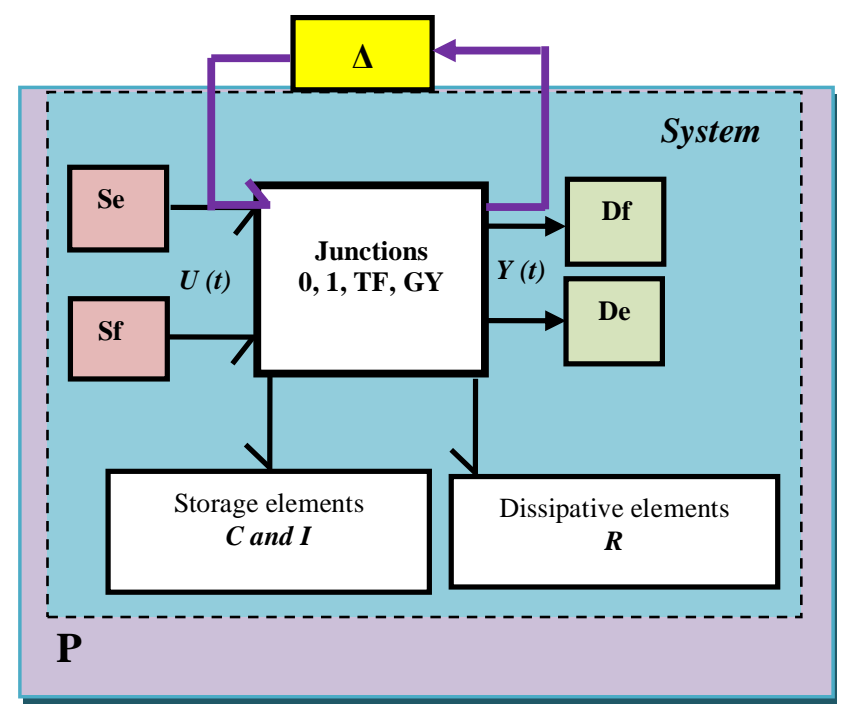

Fig.5. Representation of a BG-LFT

\section{3. Robust Residue Determination}

To determine the robust residuals of an industrial system by the bond graph approach, the following steps must be followed: 
- $1^{\text {st }}$ step: Determine the model of the industrial system by the bond graph approach using an Fractional linear transformations system;

- $2^{\text {nd }}$ step: Determine the residual equations by the redundant analytical relations (RRAs) using the LFT bond graph approach:

$\checkmark \quad$ For a junction 0:

$\sum a_{i} f_{i n c}+\dot{\sum} S f+\sum w_{i}$

$\checkmark \quad$ For a junction 1:

$\sum a_{i} e_{i n c}+\sum S e+\sum w_{i}$

$-\mathrm{a}= \pm 1$ denotes the direction of the arrow;

-0 : denotes the sum of the flux sources;

-1 : refers to the sum of the effort sources of effort.

- $3^{\text {th }}$ step: After elimination of the unknowns, the residual equations will be defined as follows:

ARRs : $\Phi\left(\sum S e, \sum S f, D e, D f, \sum w_{i}, R_{n}, I_{n}, C_{n}, T F_{n}, G Y_{n}\right)$

Or:

- TFn and Gyn are nominal elements $T F$ and $G Y$;

- $R n, C n$ and $I n$ are nominal elements $R, C$ and $I$;

- $\sum w_{i}$ is the sum of modulated inputs corresponding to uncertainties on the junction-related items.

\section{Hydraulic System by Bond graph}

\subsection{Hydraulic System}

Study of the configuration with two reservoirs We are interested here in the modeling and the monitoring of the system comprising the two reservoirs $C_{1}$ and $C_{2}$, the two level detectors $D e_{1}$ and $D e_{2}$, and two valves on / off type $R_{1}$ and $R_{2}$ (figure 6).

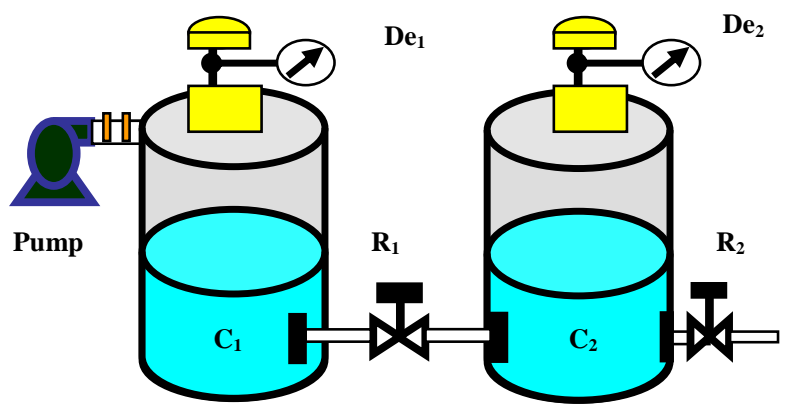

Fig.6: Hydraulic system with two tanks

\subsubsection{Bond Graph Model}

The both physical quantities characterizing the hydraulic system are the flow and pressure which correspond to the flow and effort in terminology bond graph. Using the bond graph methodology, the various elements of the system are modelled as follows (figure 7):

- The pump is modelled by a flow source $S f$;

- The tanks are modelled by storage-elements $C$;

- The valves are modeled by restriction elements $R$;

- The various connections between components system are modelled by " 0 " junctions in the case of equal pressure and "1" junctions in the case of equal flow.

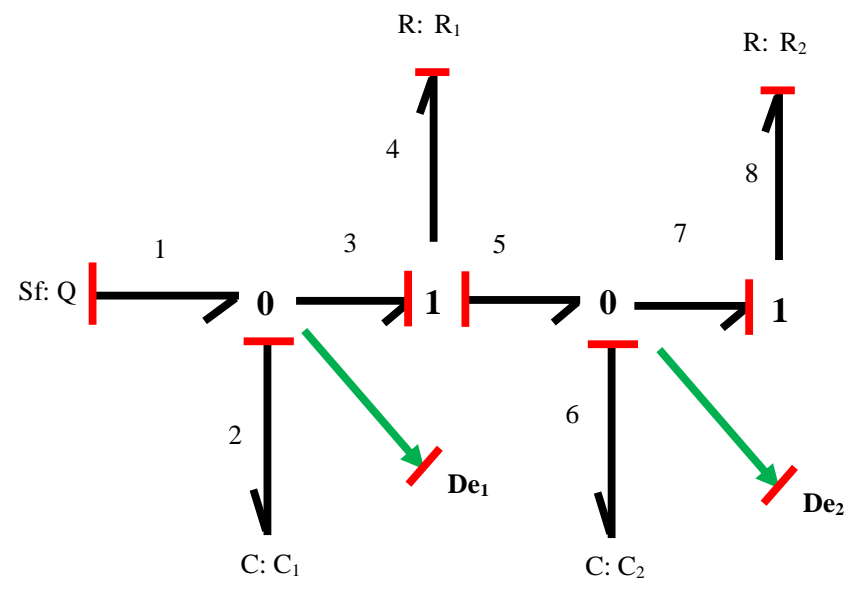

Fig.7: Bond graph model of the hydraulic system

\subsubsection{FDI by Bond Graph Modelling}

In table 1 , shown below, it is given the structural equations deduced from bond graph modelling of process (figure 7). By combining the equations presented in table 1 to eliminate unknown variables, we can generate the set of residuals in which the appeared variables (from sensors actuators) are all known.

Table 1: Structural Equations or Normal Mode

\begin{tabular}{|c|c|c|}
\hline $\mathbf{N}$ & Tunction & Structural equations \\
\hline 1 & Junction $0_{1}$ & $\begin{array}{c}f_{1}-f_{2}-f_{3}=0 \\
e_{1}=e_{2}=e_{3}=D e_{1}\end{array}$ \\
\hline 2 & Junction $0_{2}$ & $f_{5}-f_{6}+f_{7}=0$ \\
& & $e_{6}=e_{7}=e_{8}=D e_{2}$ \\
& & \\
3 & Junction $1_{1}$ & $f_{3}=f_{4}=f_{5} ;$ \\
& & $e_{3}-e_{4}-e_{5}=0$ \\
\hline 4 & Junction $1_{2}$ & $f_{7}=f_{8} ;$ \\
& & $e_{7}-e_{8}=0$ \\
\hline
\end{tabular}

For example, the junction $0_{1}$ equation as follows:

$r_{1}=f_{1}-f_{2}-f_{3}$

By replacing the flow fi by its expression deduced by its behaviour equation (generated from the BG) components, allows to write the residual $\mathrm{r} 1$ as:

$$
r_{1}=Q_{1}-C_{1} \frac{d D e_{1}}{d t}-\frac{\left(D e_{1}-D e_{2}\right)}{R_{1}}
$$

The equation (4) shows the residual $r_{1}$ is are sensitive to elements $\left(Q, C_{l}, D e_{1}, D e_{2}\right.$ and $\left.R_{l}\right)$. Consequently, when fault is occurred in each elements described above, the residual becomes different of zero $r_{l}$.

The junction $\mathrm{O}_{2}$ gives us as equation:

$$
r_{2}=f_{5}-f_{6}-f_{7}
$$

According to these relations, one can deduce the residual equation $r_{2}$ :

$$
r_{2}=\frac{\left(D e_{1}-D e_{2}\right)}{R_{1}}-C_{2} \frac{d D e_{2}}{d t}-\left(\frac{D e_{2}}{R_{2}}\right)
$$

The set of residual is grouped in the table 2 . We obtain a boolean matrix. The columns are associated to the residuals $r_{1}$ and $r_{2}$ and the lines are the boolean signatures of the monitored components. 
Table 2: Signature Faults for Normal mode

\begin{tabular}{|l|l|l|}
\hline & $r_{1}$ & $r_{2}$ \\
\hline$Q$ & 1 & 0 \\
\hline$C_{1}$ & 1 & 0 \\
\hline$C_{2}$ & 0 & 1 \\
\hline$R_{1}$ & 1 & 1 \\
\hline$R_{2}$ & 0 & 1 \\
\hline$D e_{1}$ & 1 & 1 \\
\hline$D e_{2}$ & 1 & 1 \\
\hline
\end{tabular}

The lines of the table below show the sensitivity of each residual for each element. For example, when a fault is occurred in the tank $C_{l}$, only the residual $r_{l}$ is sensitive.

\subsubsection{Simulation of the system in normal operation}

Figures 8.a, 8.b and 8.c show that the pressure at the reservoirs $C_{I}$ and $C_{2}$ evolves according to the increase of the level in $C_{l}$, and reaches its maximum when the tanks will be full.

Figure 8.c shows that, in the case of normal operation, the values average residues are almost zero.

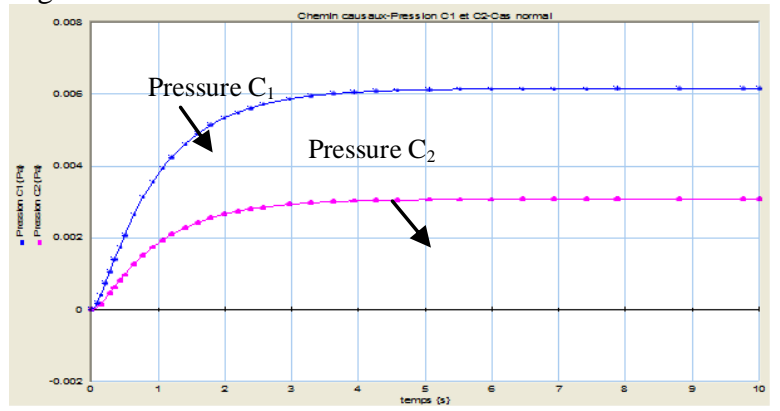

a)

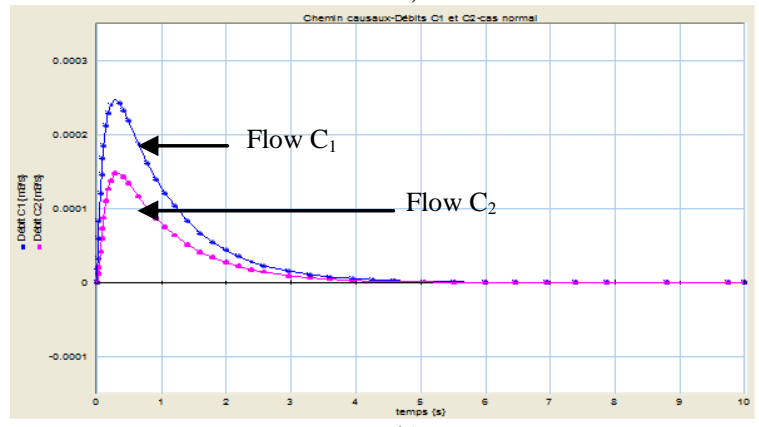

b)

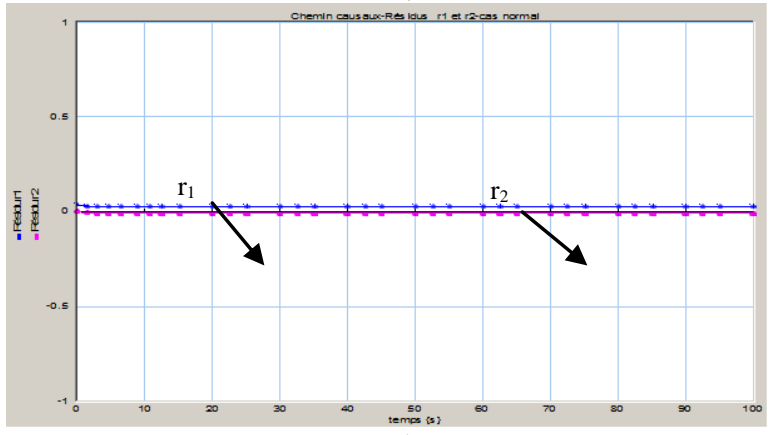

c)

Fig.8: a) Pressures, b) Flow rates, c) Residues in the case of normal operation

\subsubsection{Simulation of the System In Case Of Faults}

$>$ Valve faults (Valve blocked)

Figures 9.a, 9.b and 9.c show that when valve $R_{l}$ is blocked, the patterns of pressures and flow rates at $C_{l}$ remain unchanged while at $C_{2}$ become zero. The patterns of the residues $r_{1}$ and $r_{2}$ when the valve $R_{l}$ is plugged has non-zero average values, hence these residues are sensitive to the variation of the flow at the valve $R_{1}$, this is confirmed by the theoretical results presented in table 2 :

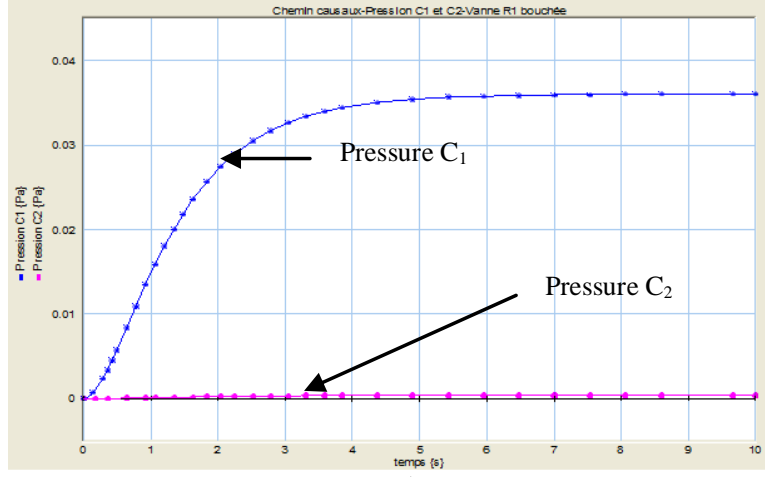

a)

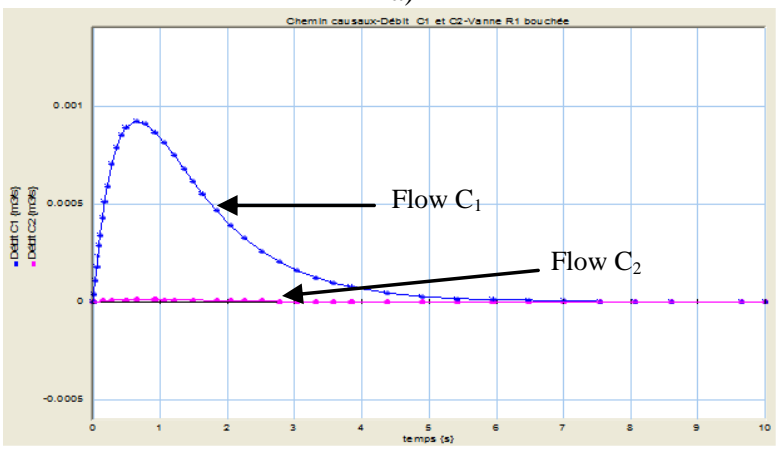

b)

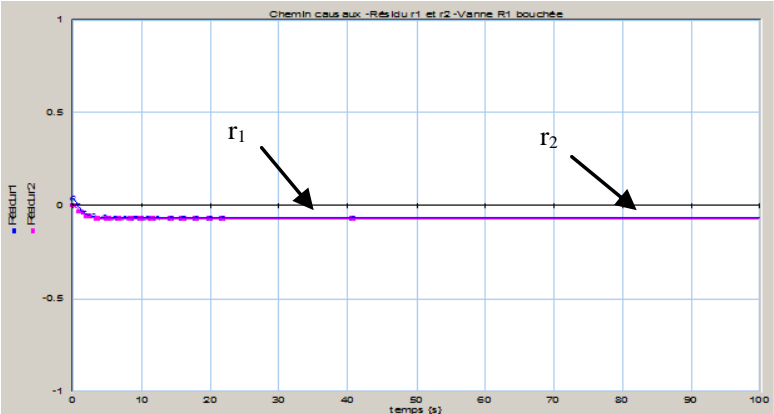

c)

Fig.9: a).Pressure, b) Flow rates, c) Residues in the event of a valve fault: valve $\mathrm{R} 1$ blocked

\section{Reservoir defects (leakage at tank C1)}

When a leak appears between the times $40 \mathrm{~s}$ and $50 \mathrm{~s}$ at the tank $C_{l}$, these pressures and flow rates (figures 10.a and 10.b decrease when the leak occurs). The residues are disturbed at the time of the occurrence of the defect; they are sensitive to this fault.

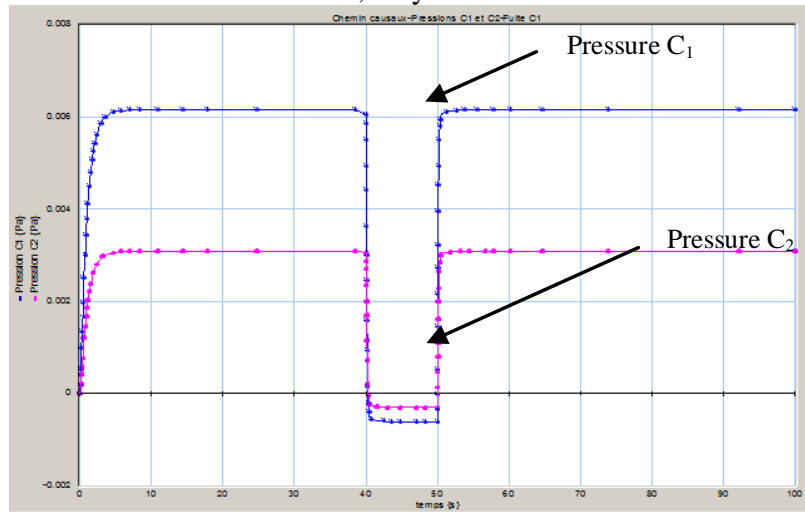




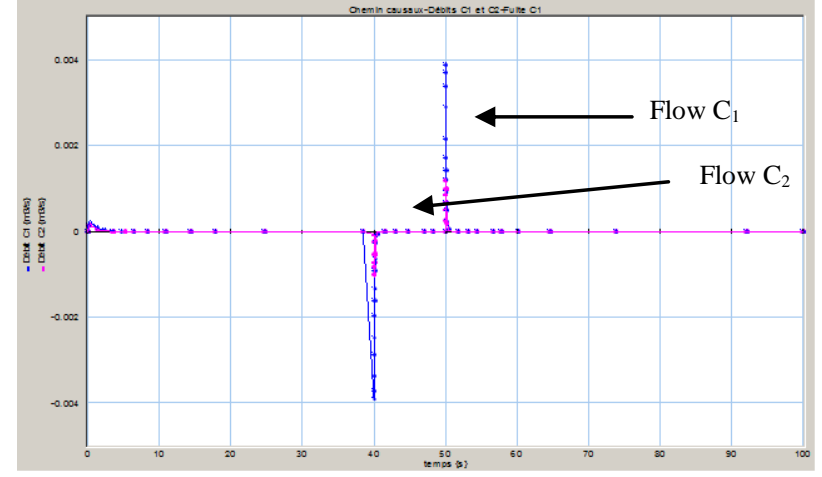

b)

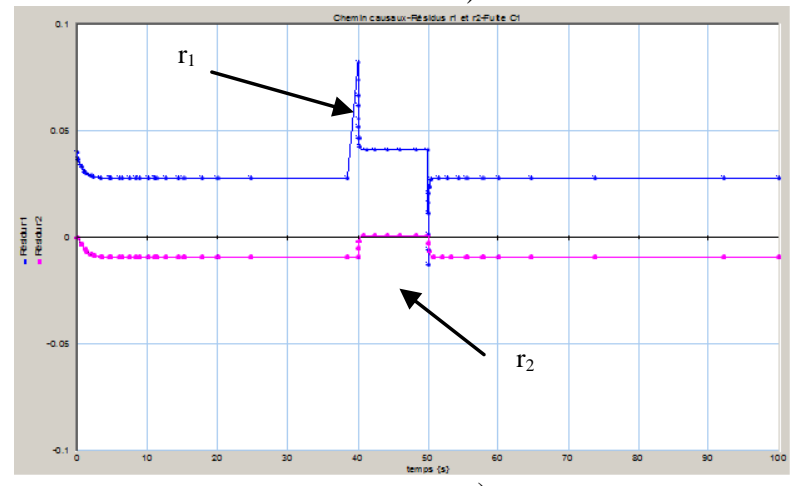

c)

Fig.10: a).Pressure, b) Flow rates, c) Residues in the event of a reservoi defects: leakage at tank $C_{l}$

\subsection{Robust Diagnosis by Bond Graphs}

Figure 11 shows the BG-LFT model of the hydraulic system.

For example, the junction 01 gives us as equation:

$R_{d l}=f_{1}-f_{2}-f_{3}+w_{I / R I}+w_{C l}$

According to these relations, one can deduce the residual equation $R d_{l}$ :

$$
R_{d l}=Q_{1}-C_{1} \frac{d D e_{1}}{d t}-\frac{\left(D e_{1}-D e_{2}\right)}{R_{1}}+w_{\frac{1}{R_{l}}}+w_{\frac{1}{C_{l}}}
$$

The equation consists of two parts: the first part is the normal evolution of the residual $r_{l n}$ and the second part represents the residual uncertainty related to the evolution of the parameters $d_{l}$ :

$$
\left\{\begin{array}{l}
R_{d 1}=r_{1 n}+d_{1} \\
r_{1 n}=Q_{1}-C_{1} \frac{d S S e_{1}}{d t}-\frac{\left(S S e_{1}-S S e_{2}\right)}{R_{I n}} \\
d_{1}=Y_{S 1}+w_{1 / R 3}+w_{C 1}
\end{array}\right.
$$

The junction 02 gives us as equation:

$$
R d_{2}=f_{5}-f_{6}-f_{7}+w_{1 / R 2}+w_{C 2}
$$

According to these relations, one can deduce the residual equation $R d_{2}$ :

$$
R d_{2}=\frac{\left(D e_{1}-D e_{2}\right)}{R_{1}}-C_{2} \frac{d D e_{2}}{d t}-\left(\frac{D e_{2}}{R_{2}}\right)+w_{\frac{1}{R_{2}}}+w_{C 2}
$$

The equation consists of two parts: the first part is the normal evolution of the residual $r_{2 n}$ and the second part represents the residual uncertainty related to the evolution of the parameters $d_{2}$ :

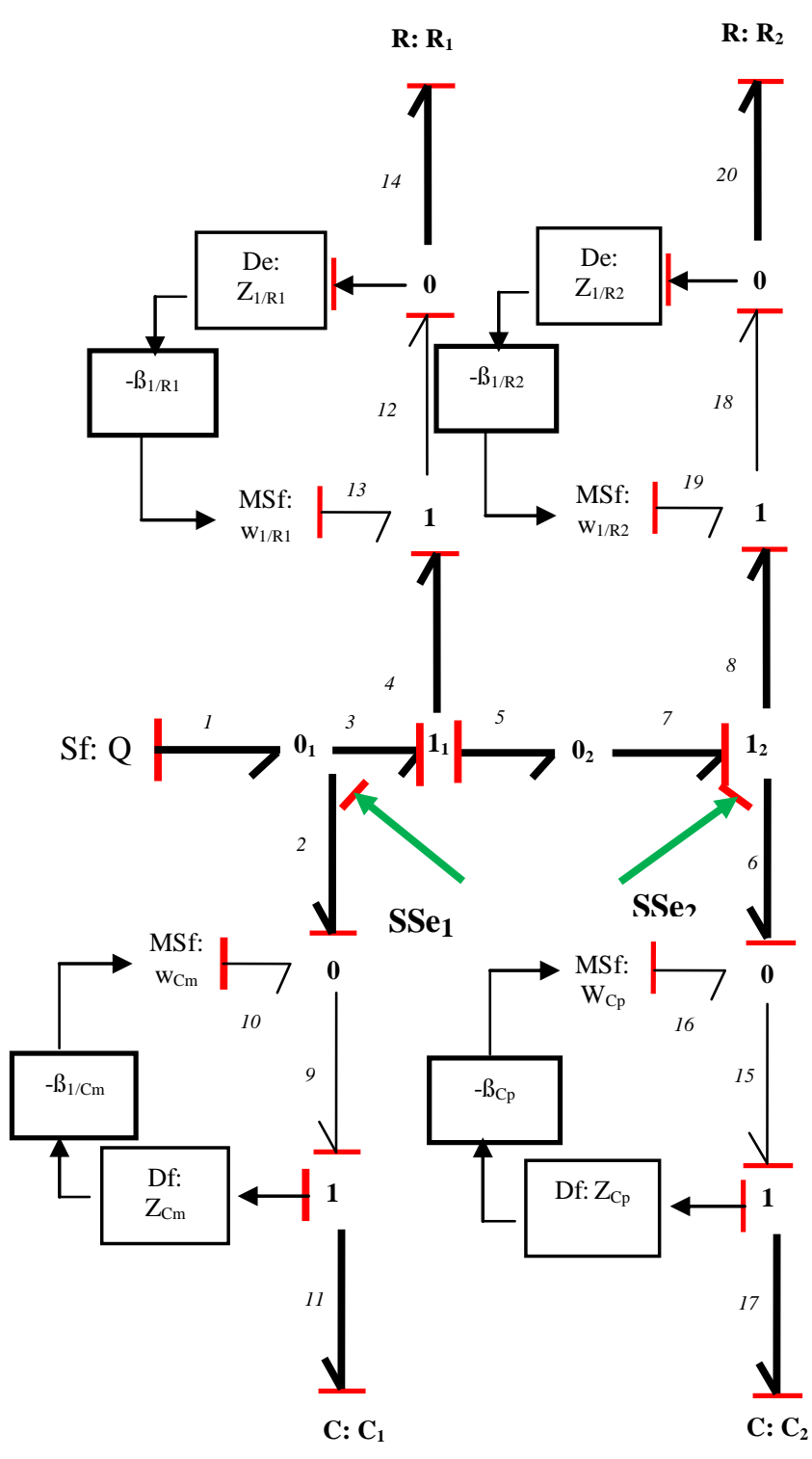

Fig.11: BG-LFT approach hydraulic system with sensors dualised

\section{Conclusion}

In this work we used the bond graph methodology for modeling, robust diagnostics and simulation of an industrial level control process. The bond graph tool gave us a great contribution in our work, on the one hand allowed us to visualize the dynamic evolution of each component of the process in the normal case and in the abnormal case, on the other hand it facilitates the robust diagnosis of the system in question by determining the normal residues separated from these uncertainties.

The figures in this article are determined by the 20SIM software dedicated to the model bond graph [29].

Future work will be on the use of the bond graph tool for building a Luenberger observer to determine state-back control

\section{Acknowledgement}

This contribution is the result of the research on electrical systems using the bond graph approach: this research is determined at the laboratory Analysis, Design and Control Systems LaboratoryENIT, Faculty of Sciences of Tunis, Tunis El Manar University, Tunis, Tunisia.

$$
\left\{\begin{array}{l}
R_{2}=r_{21 n}+d_{2} \\
r_{2 n}=\frac{\left(D e_{1}-D e_{2}\right)}{R_{1}}-C_{2} \frac{d D e_{2}}{d t}-\left(\frac{D e_{2}}{R_{2}}\right) \\
d_{2}=+w_{\frac{1}{R_{2}}}+w_{C 2}
\end{array}\right.
$$




\section{References}

[1] S. Benmoussa, B. Ould-Bouamama, R. Merzouki. "Bond Graph Approach for Plant Fault Detection and Isolation: Application to Intelligent Autonomous Vehicle". Automation Science and Engineering, IEEE Transactions on, vol.11, no.2, pp.585-593, April 2014.

[2] R. Loureiro, R. Merzouki and B. Ould Bouamama. "Bond Graph Model Based on Structural Diagnosability and Recoverability Analysis: Application to Intelligent Autonomous Vehicles". IEEE Transactions on Vehicular Technology, vol. 61, $\mathrm{N}^{\circ} 3$, pp. 986-997, March 2012.

[3] T. K. Bera, R. Merzouki, B. Ould Bouamama and A.K. Samantaray. "Design and validation of a reconfiguration strategy for a redundantly actuated intelligent autonomous vehicle". Journal of Systems and Control Engineering vol. 226, n 8 , pp. 1060-1076, sept. 2012.

[4] Y. Touati, R. Merzouki and B. Ould Bouamama. "Robust diagnosis to measurement uncertainties using bond graph approach: Application to intelligent autonomous vehicle" Mechatronics, Publisher (Elsevier), Vol. 22, Issue 8, pp. 1148-1160, Dec. 2012.

[5] A.K. Samantaray, and B. Ould Bouamama, "Model-based process supervision. A bond graph approach," Springer Verlag, Series: Advances in Industrial Control, 2008.

[6] B. Ould Bouamama, K.Medjaher, M. Bayart, A.K, Samantary, and B Conartd, "Fault detection and isolation of smart actuators using bond graphs and external model,". Control Engineering Practice 13, 159-175, 2005

[7] M. Kamal, "Contribution de l'outil bond graph pour la conception de systèmes de supervision des processus industriels,". Thèse doctorat, Université des Sciences et Technologies de Lille, 2005.

[8] J. Thoma et B. Ould Bouamama : Modelling and simulation in thermal and chemical engineering. Bond graph approach, SpringerVerlag, 2000.

[9] B. Ould Bouamama, G. Dauphin-Tanguy, M. Staroswiecki, F Buison, "Bond graph technique as a decision-making tool in supervision system", HKK Conference \& Symposium in Graph Theoretic \& Entropy Methods in Engineering, University of Waterloo, June 13-15, pp. 91- 97, 1999.

[10] M. Staroswiecki. M. Bayart, "Models and languages for the interoperability of smart instruments". Automatica, Vol. 32, No. 6. pp. 859-873, 1996

A. Achir : Contribution à l'étude de la propriété de Platitude sur des modèles bond graphs non linéaires, Thèse de Doctorat université de Lille, 2005.

[11] M. Vergé, D. Jaume, "Modélisation structurée des systèmes avec les bond graphs,". Edition Technip, Paris, 2004.

[12] G. Dauphin-Tanguy, "Les bonds graphs," edition Hermès, 2000

[13] M. Mosiek, "Procédures graphiques pour l'analyse structurelles de systèmes physiques modélisés par bond graph ". Thèse de doctorat, Université des Sciences et Technologies de Lille, 2000.

[14] M. Tagina, J. P. Cassar, G. Dauphin-Tanguy, M. Staroswiecki, "Monitoring of systems modelled by bond graph,", ICBGM'95, International Conference on Bond Graph Modelling. Las Vegas, pp.275-280, 1995

[15] H.M. Paynter. « Analysis and design of engineering systems», M.I.T.Press, 1961

[16] D.C. Karnopp. and R.C. Rosenberg. « Systems Dynamics: a Unified Approach », Mac Graw Hill, 1983.

[17] R.C. Rosenberg. «Introduction to physical System Dynmics», Series in mechanical engineering, Mac Graw Hill, 1983.

[18] M. Tagina. and G. Dauphin, Tanguy. « La méthodologie bond graph. Principes et applications», Centre de Publication Universitaire, 2003.

[19] M. Kamal.”Contribution de l'outil bond graph pour la conception de systèmes de supervision des processus industriels", Thèse doctorat, Université des Sciences et Technologies de Lille, 2005.

[20] M. Mosiek. "Procédures graphiques pour l'analyse structurelles de systèmes physiques modélisés par bond graph", Thèse de doctorat, Université des Sciences et Technologies de Lille, 2000.

[21] A.K. Samantaray and B. Ould Bouamama. B.”Model-based process supervision. A bond graph approach", Springer Verlag, Series: Advances in Industrial Control, 2008.

[22] B. Ould Bouamama, K. Medjaher, K. Bayart, A.K. Samantaray, and B. Conrard. "FDI of Smart actuators using Bond Graphs and external models". Control Engineering Practice, vol. 13, n², pp. 159175. 2005.

[23] Sié Kam. C.’'Les Bond Graphs pour la Modélisation des Systèmes Linéaires Incertain", Thèse de doctorat. USTLille1-ECLille. Décembre 2001. N॰ d'ordre 3065, 2001.
[24] M. A. Djeziri. 'Diagnostic des Systèmes Incertains par l'Approche Bond Graph", Thèse de Doctorat École Centrale de Lille 2007.

[25] R. Redheffer. "On a certain linear fractional transformation", EMJ. Maths and phys. 39, pp. 269-286, 1960.

A. Oustaloup. "La robustesse", Hermès ISBN. 2.86601.442.1, 1994.

[26] D. Alazard, C. Cumer, P. Apkarian, M. Gauvrit, G. Fereres."Robustesse et Commande Optimale", Cépadues-Editions ISBN. 2.85428.516.6, 1999.

[27] Controllab Products, 20-sim version 4.0, http://www.20sim.com. 